\title{
Skin microbiome and acne vulgaris: Staphylococcus, a new actor in acne
}

\author{
Brigitte Dreno $^{1,2}$ | Richard Martin ${ }^{3}$ | Dominique Moyal ${ }^{5}$ | Jessica B. Henley ${ }^{4}$ | \\ Amir Khammari $^{1,2}$ | Sophie Seité ${ }^{5}$ (D)
}

${ }^{1}$ Department of Dermatology, Nantes University Hospital, Nantes, France

${ }^{2} \mathrm{CIC}$, Inserm U892-CNRS 6299, Nantes, France

${ }^{3}$ L'Oréal Research and Innovation, Tours, France

${ }^{4}$ Cooperative Institute for Research in Environmental Sciences, University of Colorado, Boulder, CO, USA

${ }^{5}$ La Roche-Posay Dermatological Laboratories, Asnières, France

\section{Correspondence}

Sophie Seité, La Roche-Posay Dermatological Laboratories, Asnières, France.

Email: sophie.seite@loreal.com

Funding information

La Roche-Posay Dermatological Laboratory.

\begin{abstract}
Propionibacterium acnes (P. acnes), the sebaceous gland and follicular keratinocytes are considered the three actors involved in the development of acne. This exploratory study investigated the characteristics of the skin microbiota in subjects with acne and determined microbiota changes after 28 days of application of erythromycin $4 \%$ or a dermocosmetic. Skin microbiota were collected under axenic conditions from comedones, papulo-pustular lesions and non-lesional skin areas from subjects with mild to moderate acne according to the GEA grading using swabs. Samples were characterized using a high-throughput sequencing approach that targets a portion of the bacterial 16S rRNA gene. Overall, microbiota samples from 26 subjects showed an overabundance of Proteobacteria and Firmicutes and an under-representation of Actinobacteria. Staphylococci were more abundant on the surface of comedones, papules and pustules ( $P=.004$ and $P=.003$ respectively) than on non-lesional skin. Their proportions increased significantly with acne severity $(P<.05$ between GEA-2 and GEA-3). Propionibacteria represented less than $2 \%$ of the bacteria on the skin surface. At Day 28, only the number of Actinobacteria had decreased with erythromycin while the dermocosmetic decreased also the number of Staphylococci. A significant reduction $(P<.05)$ from Day 0 of comedones, papules and pustules with no significant difference between the products was observed. The bacterial diversity on all sampling areas was similar. The dermocosmetic decreased the number of Actinobacteria and Staphylococcus spp. after 28 days. Staphylococcus remained the predominant genus of the superficial skin microbiota. No significant reduction in Staphylococcus spp. was observed with the topical antibiotic.
\end{abstract}

\section{KEYWORDS}

acne, diversity, microbiome, microbiota, Propionibacterium, skin, Staphylococcus

\section{1 | INTRODUCTION}

The skin, like the gut and other body tissues, is colonized by a dense community of commensal microorganisms. This symbiotic relationship between the skin and the commensal microbial community, the microbiota, forms a complex barrier against external insults with differences between the microbiome of the skin surface and skin appendices. ${ }^{[1]}$
The colonizing microorganisms are in a constant dialogue with their host by the virtue of complex signals provided by the innate and the adaptive immune systems. This mutualistic relationship leads to a well controlled but delicate equilibrium, the microbiome, which is mandatory for healthy skin. ${ }^{[1]}$ On the skin, four main bacterial phyla have been identified, Actinobacteria, Firmicutes, Proteobacteria and Bacteroidetes. The three most commonly observed genera are 
Corynebacteria, Propionibacteria and Staphylococci. ${ }^{[2]}$ Changes in the natural composition of cutaneous microbial communities, such as loss of diversity have been linked to chronic inflammatory skin diseases, including atopic dermatitis, psoriasis and acne. ${ }^{[3-6]}$

Acne is a chronic inflammatory disease affecting the sebaceous unit. Three main factors are involved in the development of acne: (i) increased sebum production caused by the stimulation of the sebaceous gland via the activation of several receptors including those for androgens, neuropeptides, insulin-like growth factor-I and peroxisome proliferator-activated receptors (PPAR), (ii) abnormal keratinization of the sebaceous duct and comedone formation and (iii) an inflammatory immune response in which Propionibacterium acnes (P. acnes) and the innate immunity play an important role. ${ }^{[7,8]}$

The bacterium is known for triggering proinflammatory cytokine release and expression of antimicrobial peptides. Overcolonization of $P$. acnes causes activation of monocyte Toll-like receptor 2 (TLR2), resulting in the production of Interleukin-12 (IL-12) and IL-8. ${ }^{[9]}$ IL-12 is the major proinflammatory cytokine produced by monocytes in response to invading Gram-positive organisms. ${ }^{[9,10]}$ A certain amount of data has shown that different phylotypes of $P$. acnes activate the innate immunity. This is probably why these phylotypes may play a more determinant role in the acne lesion severity than actually the intensity of their proliferation. ${ }^{[11,12]}$ Moreover, genome comparison of different $P$. acnes strains identified different commensal P. acnes subtypes between skin areas without and with acne lesions. ${ }^{[13]}$ That is why the severity of acne may be more related to the selection of its subtypes than to its proliferation.

Furthermore, excessive sebum production, which is currently associated with the development of acne lesions and which is characterized by both an increased production and a qualitative modification of sebum, may play a role in the selection of the subtype of $P$. acnes. ${ }^{[14,15]}$

Even though the association between $P$. acnes and acne vulgaris is well established, very few studies have investigated the entire facial skin microbiota of patients with acne. Three-dimensional topographic analyses and microbiome profiling have shown differences between the microbiota composition in healthy skin and in skin with acne, as well as natural differences in microbial colonization between the sebaceous gland and the skin surface. ${ }^{[16]}$ Moreover, cutaneous bacterial communities have been shown to be involved in the immune homoeostasis and inflammatory responses; and both are known for triggering acne. $^{[17]}$

But, P. acnes is not only one of the main acne triggers, it is also a commensal bacterium inhabiting the sebaceous follicle. As such, it plays a physiological role in inhibiting the invasion of pathogenic bacteria such as Staphylococcus aureus (S. aureus) and S. pyogenes in making the skin inhospitable for these pathogens such as $S$. aureus or Streptococcus pyogenes while allowing other commensal Staphylococci strains such as S. epidermis to grow. ${ }^{[1,18,19]}$ P. acnes maintains the natural $\mathrm{pH}$ in the skin and that of the sebaceous glands by hydrolyzing triglycerides, releasing free fatty acids and secreting propionic acid. ${ }^{[1]}$ Moreover, Staphylococcus epidermidis and P. acnes have been shown to interact. ${ }^{[20]}$ Studies suggest that $S$. epidermidis owns an arsenal of different mechanisms to inhibit proliferation of $P$. acnes ${ }^{[20,21]}$ participating in the equilibrium of the microbiota and in a balanced immune system, thereby allowing for a healthy skin.

The aim of this study was to investigate the microbiota profile on the epidermis of skin areas with acne lesions (comedones and papulopustular lesions) and of skin areas without visible acne lesions. A secondary objective was to compare the change of these communities in a split-face study using an intra-individual method in acne patients receiving either a topical antibiotic or a dermocosmetic for 28 days.

\section{2 | MATERIALS AND METHODS}

\subsection{Ethics}

This single-centre, controlled, randomized, double blind, intraindividual (split face) comparative exploratory study was conducted between March and June 2014 according to Good Clinical Practices. The study received approval from the local ethics committee of Nantes on 6 November 2013 under the reference number "Référence 37/13."

Written informed consent and photography consent were obtained from each subject before enrolment.

\section{2 | Patient profile}

The study included subjects with mild to moderate acne (grades 2 and 3 on the GEA acne grading scale ${ }^{[22]}$ ) with at least 20 comedones and 10 papulo-pustular lesions equally distributed over the face. Subjects were not allowed to use a local acne treatment within 2 weeks, an oral antibiotic within 4 weeks or oral isotretinoin within 3 months prior to inclusion. Women of childbearing potential had to use reliable contraception, while breastfeeding and pregnant women were not allowed to participate in the study.

\section{3 | Products}

A topical antibiotic (4\% erythromycin, Erythrogel ${ }^{\circledR}$, Laboratoire Bailleul Biorga, France) and a dermocosmetic (Effaclar ${ }^{\circledR}$ Duo+, La Roche-Posay Laboratoire Dermatologique, Asnières, France) containing lipohydroxy acid, salicylic acid, linoleic acid, niacinamide, piroctone olamine, a ceramide and thermal spring water (TSW) were applied daily for 28 days on each half-face. Subjects were randomized at the investigational site according to a randomization plan. Products were applied by the site personnel from Mondays to Fridays; subjects applied the products at home on Saturdays and Sundays. They were instructed not to change their hygiene habits or to apply other skin care products or topical drugs during the study on their face.

\subsection{Clinical evaluations}

Clinical evaluations were conducted by the same investigator on days 0,14 and 28 and included the scoring of acne severity using the GEA grading scale, the inflammatory and non-inflammatory lesions count and the reporting of local tolerance issues, acne signs and symptoms. 


\section{5 | Microbiota sampling}

Microbiota sampling of selected facial areas was conducted at all study visits by the same investigator. Skin microbiota samples were collected on the cheeks, forehead, temple or chin of each patient's face (depending on the location of acne lesions) using aseptic techniques under sterile airflow generated by a portable hood. Single use sterile square-sized cotton-tipped swabs (165KS01, COPAN SPA, Brescia, Italy) were moistened with a sterile solution of deionized water containing $0.15 \mathrm{~mol} \mathrm{~L}^{-1} \mathrm{NaCl}$ and $0.1 \%$ Tween 20. In total, three areas on the face were sampled as follows: one with comedones, one with papulo-pustular lesions and one area without acne lesions, serving as a negative intra-individual control. The selected areas were rubbed firmly with the swabs for 20 seconds. Each sampled area of a size of $9 \mathrm{~mm}^{2}$ was identified using a positioning mask and standardized photography to ensure that the same area was sampled at each follow-up visit. Cotton tips containing the samples were stored at $-80^{\circ} \mathrm{C}$ until the end of the study and shipped for processing using dry ice to the University of Colorado, Boulder, Colorado, USA

\section{6 | DNA extraction, PCR amplification, and sequencing}

To allow for a complete microbiota profiling over time, samples from individuals with missing paired samples or with samples containing insufficient bacterial material at visits Day 14 or 28 were not considered for analysis. DNA was extracted from the swabs using the MoBio PowerSoil-htp 96-well Soil DNA Isolation Kit (MoBio, Inc., Carlsbad, CA, USA) following the manufacturer's instructions. PCR amplification was performed in triplicate for each DNA sample. ${ }^{[23]}$ DNA was PCR amplified with barcoded $515 \mathrm{~F}$ and $806 \mathrm{R}$ primers that targets the V4 region of bacterial and archaeal $16 \mathrm{~S}$ rRNA genes ${ }^{[24]}$ using the 5 PRIME Hot Master Mix (5 PRIME Inc., Bethesda, MD, USA). Negative controls were included at all steps in the sample processing to test for contamination (no sequences were recovered from these negative controls). Triplicate PCRs were pooled for each sample, and amplicon concentrations were measured with a PicoGreen dsDNA assay (Life Technologies, Grand Island, NY, USA). Amplicons were pooled by plate at equimolar concentrations for each sample and then cleaned with the UltraClean PCR Clean-Up Kit (MoBio Inc., Carlsbad, CA, USA). Cleaned pools were combined at a final yield of $2 \mu \mathrm{g}$ of DNA and sequenced on the Illumina MiSeq platform at the University of Colorado Next Generation Sequencing Facility, Boulder, Colorado, USA.

\section{7 | Sequence processing}

Sequences were processed as previously described. ${ }^{[23]}$ Sequences were demultiplexed, and forward and reverse $16 \mathrm{~S}$ rRNA gene reads were merged. All resulting sequences were quality-filtered, and singletons were removed with QIIME and UPARSE. ${ }^{[24,25]}$ Sequences were then de-replicated, and a database containing one sequence for each operational taxonomic unit (OTU) was generated using UCLUST v7 at the $97 \%$ nucleotide identity level. ${ }^{[26]}$ Sequencing reads from the full data set were then clustered to the database to generate an OTU table. Taxonomy was assigned to each OTU using the Ribosomal Database Project taxonomic classifier. ${ }^{[27]}$ OTUs represented by fewer than five reads across the entire data set and all OTUs identified as coming from mitochondria or chloroplasts were removed prior to downstream analyses. To compare all samples at equivalent sequencing depth, the OTU table was rarefied to 2.000 sequences per sample.

\section{8 | Statistical analysis of clinical data}

Statistical analysis of clinical data was performed using StatView 5.0. Continuous variables were described by means and standard deviations (SD). Qualitative variables were described by absolute numbers and corresponding percentages. Quantitative variables were compared at days 0,14 and 28 using a Wilcoxon rank sum test for paired observations and qualitative variables using a McNemar's chi-square test. To determine the differences between both products at Day 0 and Day 28 , the number of lesions was compared using the Wilcoxon rank sum test for paired samples.

\section{9 | Statistical analysis of sequence data}

The statistical analysis of sequence data was performed using R 2.15. Statistical tests were done on the raw count of rarefied samples. Bacterial populations at different taxonomical levels (genus and phylum) were compared at Day 0 and Day 28 using a Kruskal-Wallis oneway analysis of variance.

\section{3 | RESULTS}

\section{1 | Demographic and baseline data}

The study included 55 subjects with skin phototype II (45\%), III (49\%), IV (4\%) or VI (2\%) on the Fitzpatrick scale. The majority of subjects (33 subjects) were female; the mean age was $23 \pm 6$ years ranging from 15 to 43 years. The mean acne score on Day 0 was $2.31 \pm 0.47$; mean acne duration was $8 \pm 7$ years.

Skin samples from 26 subjects (11 male and 15 female, with a mean age of $24 \pm 6.5$ years, a mean GEA acne grade of $2.4 \pm 0.5$ and a mean acne history of $10 \pm 6$ years) provided a sufficient quantity of microbiota material allowing for a microbiota analysis before and after applications of the products at all time points. On average, at Day 0 , subjects had $31 \pm 7$ comedones and $19 \pm 6$ papulo-pustular lesions on their entire face (Table S1).

\subsection{Skin surface microbiota of subjects with acne}

The average number of lesions on Day 0 of each half-face is presented in Table S2. The Shannon Index indicated a similar microbial diversity on areas with papulo-pustular lesions, with comedones and skin without acne lesions; between-group differences for acne severity grade $2(n=16)$ and grade $3(n=10)$ were statistically not significant $(P=.39)$.

At Day 0 , the analysis of the bacterial phyla showed that, independently from the location ( $40 \%$ on cheeks, $6 \%$ on the forehead, $9 \%$ on the temples 


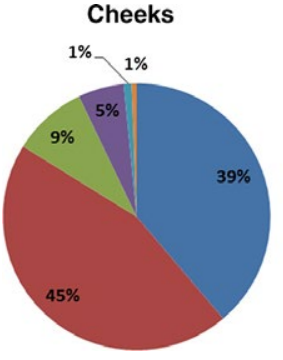

D_Firmicutes

- p_Proteobacteria

= p_Actinobacteria

a $\mathrm{p}$ _Bacteroidetes

n_Fusobacteria

m Other

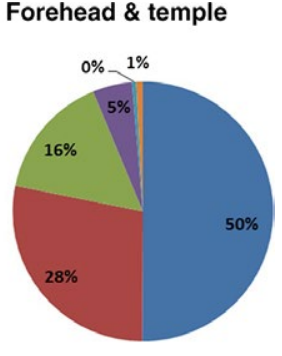

FIGURE 1 Bacterial Phyla ( $\mathrm{p}_{-}$) at the skin surface of the cheek $(n=29)$ or forehead and temple $(n=11)$ of subjects with acne at D0

or $45 \%$ on the chins), all three sampling areas had similar profiles, with no difference in acne severity. However, depending on the location of the sampling areas, different skin surface microbiota profiles were observed. On the forehead and temple, significantly more Firmicutes (50\%, $P=.042$, compared to $39 \%$ on cheeks), slightly more Actinobacteria (16\%, $P=.092$ compared to $9 \%$ on cheeks) and significantly less Proteobacteria (28\%, $P=.005$, compared to $45 \%$ on cheeks) were found, see Figure 1.

Moreover, independently from the location on the face, Proteobacteria were significantly less abundant in areas with comedones and papulo-pustular lesions than in areas without acne lesions (29\% vs $34 \% ; P=.001$ and $31 \%$ vs $34 \% ; P=.05$, respectively for acne lesions and areas with no acne lesions), while Firmicutes were significantly more abundant in areas with comedones compared to areas without acne lesions ( $52 \%$ vs $47 \%-P=.002$ ); no difference was observed for Actinobacteria (Figure 2).

Table 1 provides results for the main bacterial phyla and genus of the three sampled areas of the 26 subjects at Day 0 . Staphylococci were the most abundant bacteria gender on the skin surface $(>27 \%$ of all bacteria) and were significantly more abundant on acne lesions than on areas without acne lesions (33.9\% and 34.0\% for comedones and papulo-pustular lesions vs $26.8 \%$ skin areas without acne lesions, $P<.05)$; their number increased with the severity of the condition
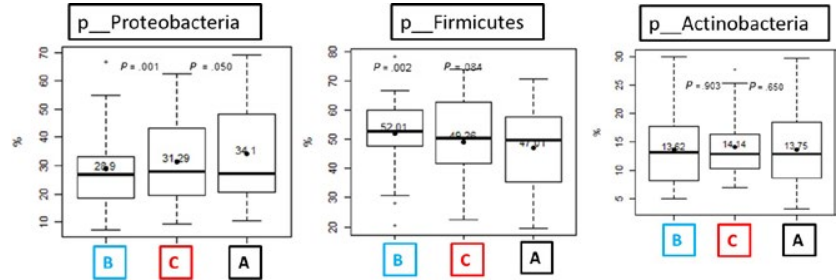

FIGURE 2 Boxplots of main bacterial Phyla (p_) on the skin surface of the three sampled areas (comedones (B), papulo-pustular (C) and skin are with no acne lesions (A) at Day $0(n=26))$-Test of difference of mean between $B$ vs $A$ and $C$ vs $A$ by main bacterial Phyla

(Figure 3). Conversely, Propionibacteria represented less than $2 \%$ of the total number of bacteria in all sampled areas.

\section{3 | Skin surface microbiota of subjects with acne after application of the topical products}

At Day 28, analysis of the main bacterial phyla and genus of the three sampled areas showed that erythromycin reduced the number of Actinobacteria while the dermocosmetic reduced both the number of Actinobacteria and Staphylococcus spp.; Tables S3 and S4 provide further information.

After 28 days, the number of both non-inflammatory and inflammatory lesions had significantly decreased $(P<.05)$ with no significant difference between the tested products (Figure S1).

Both products were well tolerated with no signs of erythema, dryness, desquamation, pruritus, stinging or burning sensation.

\section{4 | DISCUSSION}

The aim of this exploratory study was to investigate the characteristics of the microbiota on the surface of both skin areas without and with

TAB LE 1 Main bacterial phyla and genus in percentage at the skin surface of the 3 sampled areas (comedones, papulo-pustular and skin with no acne lesions) at Day $0(n=26)$

\begin{tabular}{|c|c|c|c|c|}
\hline Phylum & Genus & \multicolumn{3}{|l|}{ Area } \\
\hline \multirow[t]{3}{*}{ Actinobacteria } & & 13.61 & 14.15 & 13.75 \\
\hline & Propionibacterium & 1.04 & 1.20 & 1.36 \\
\hline & Corynebacterium & 7.93 & 8.54 & 7.71 \\
\hline \multirow{2}{*}{ Firmicutes } & Staphylococcus & $33.87^{*}$ & $34.00^{*}$ & 26.85 \\
\hline & Other Firmicutes & 18.14 & 15.27 & 20.16 \\
\hline Proteobacteria & & $28.90^{*}$ & $31.30^{*}$ & 34.10 \\
\hline Bacteroidetes & & 4.16 & 3.78 & 3.73 \\
\hline
\end{tabular}

${ }^{*} P<.05$ vs skin with no acne lesions. 


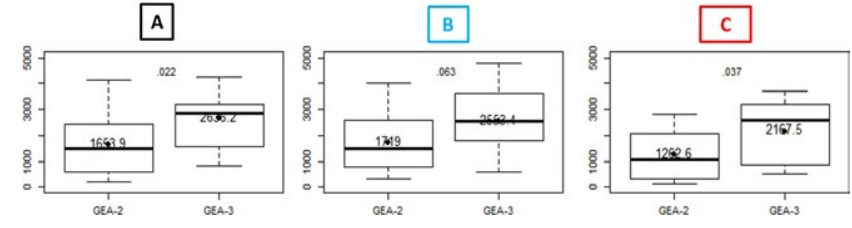

FIGURE 3 Boxplots of Staphylococcus genus at the skin surface of three sampled areas (comedones (B), papulo-pustular area (C) and skin area with no acne lesions (A) with GEA-2 ( $n=16)$ and GEA-3 acne severity $(n=10)$ at Day 0 -test of difference of mean between GEA-2 vs GEA-3 by area

acne lesions and to determine changes in the microbiota profile after 28 days of a once-daily application of either erythromycin $4 \%$ or a dermocosmetic containing lipohydroxy acid, salicylic acid, linoleic acid, niacinamide, piroctone olamine, a ceramide and thermal spring water.

The study showed that prior to the application of the products, the skin surface microbiota of the different sampled areas was dominated by Staphylococcus, while Propionibacteria represented less than $2 \%$ of the population. These results contrast with data reporting that in subjects with no acne Propionibacteria represents more than $30 \%$ of the facial microbiota. ${ }^{[2,28]}$ Moreover, the study showed that in subjects with acne, the bacterial diversity of the skin microbiota was similar, regardless the sampling area.

The present study also confirmed previously reported observations that different microenvironments may play, due to their different $\mathrm{pH}$ (4.75-5.04 for the forehead and 4.2-5.9 for the cheek in healthy subjects) and temperature $\left(33.4^{\circ} \mathrm{C}\right.$ for the forehead and 31.8 for the cheek) and also due to the different levels of sebum production (higher on the forehead than on the cheek), a role in the growth or inhibition of microorganisms. ${ }^{[20,29,30]}$

It has been acknowledged that in patients with acne, the microbiota of the sebaceous follicle is predominantly inhabited by P. acnes. ${ }^{[1,30,31]}$ As a counterpart, our results suggest that the skin surface is dominated by Staphylococci and especially S. epidermidis. This distribution may be due to the different characteristics of the two bacteria: $P$. acnes is an anaerobic while $S$. epidermidis is an aerobic and facultative anaerobic bacteria able to grow in an aerobic environment using fermentation as a defense, thus inhibiting $P$. acnes growth as reported recently by Wang et al. ${ }^{[21]}$ But, S. epidermidis does not only use fermentation to regulate $P$. acnes growth. A genome comparison made by Christensen et al. underlined the diversity of S. epidermidis and detected multiple clade- or strain-specific mobile genetic elements encoding a variety of functions important in antibiotic and stress resistance, biofilm formation and interbacterial competition, including bacteriocins such as epidermin. Moreover, the authors isolated one species with an antimicrobial activity against $P$. acnes harbouring a functional ESAT- 6 secretion system that might be involved in the antimicrobial activity against $P$. acnes via the secretion of polymorphic toxins. ${ }^{[20]}$ Finally, Xia et al. showed that S. epidermidis inhibits P.acnes-induced inflammation in skin. Staphylococcal lipoteichoic acid activated TLR2 to induce miR-143 in keratinocytes, and miR-143, in turn, directly targeted 3' UTR of TLR2 to decrease the stability of TLR2 mRNA and decreased the TLR2 protein, thus inhibiting $P$. acnes-induced pro-inflammatory cytokines. ${ }^{[32]}$
The presently reported results may add further evidence confirming that Propionibacteria and Staphylococci interact. This interaction results in variations of the microbiota of acne lesions of different zones of the body such as the cheek and forehead. This finding is in line with observations made by Zeeuwen et al. that drier body sites predominantly host Staphylococcus, Propionibacterium, Micrococcus, Corynebacterium, Enhydrobacter and Streptococcus genus. ${ }^{[33]}$ Unfortunately, the very small sample surface, corresponding to the size of an acne lesion, limited the number of usable samples and did not allow to determine the different Staphylococci species. Only an analysis of the skin microbiota comparing samples from a more important surface, obtained from the same patient, using both swabs for sampling the skin surface microbiota and strips sampling the skin surface and follicle microbiota may allow for a better differentiation between the bacterial population of the follicle and the skin surface.

Moreover, the present results indicate that the concentration of Staphylococcus increases with the severity of acne, which contradicts observations made by Numata et al. in 2014 who reported that there are no significant differences in density between Propionibacteria and Staphylococci populations. ${ }^{[34]}$

To date, the role of $S$. epidermidis in acne remains to be elucidated, while the role of Propionibacteria in the development of acne lesions is considered as predominant with a great majority of acne treatments continuing to target this bacterial genus exclusively, using their antibacterial effects as a main argument. ${ }^{[35,36]}$

Topical antibiotics in monotherapy to manage acne are not recommended anymore because of the confirmed development of antibiotic resistance, especially to macrolides. ${ }^{[37,38]}$

Despite the antibacterial resistance issue, we felt that using one of the most currently used topical macrolides, erythromycin, without concomitantly using other topical acne treatments such as retinoids or benzoyl peroxide, which might have potentially interfered with the chosen products, was the most suitable approach to assess the antibacterial benefit of the tested dermocosmetic.

The study demonstrated that erythromycin reduced the number of Actinobacteria (including Corynebacterium and Propionibacterium) while it only had a limited antibacterial effect on Staphylococci, potentially confirming the increased resistance of the bacterium to macrolides. ${ }^{[39,40]}$ But, the tested dermocosmetic not only reduced the number of Actinobacteria-it also reduced the number of Staphylococcus spp. However, it remained the predominant genus of the superficial skin microbiota of acne lesions as well as of that with no acne lesions.

The present results confirm the antibacterial benefit of the tested dermocosmetic on both Actinobacteria and Staphylococcus spp. The tested dermocosmetic may be a potential alternative to topical macrolides in the management of acne that bears the advantage of not causing antibacterial resistance of the targeted bacteria.

\section{ACKNOWLEDGEMENTS}

Le Dantec G. and Fortuné M. provided technical support. Statistical analyses were performed by Morineau A. and Huynh T.M.T. Göritz P., SMWS, France, provided editing support. Dreno B., Martin R., Moyal D., Henley J.B., Khammari A. and Seité S. all contributed to the interpretation 
and analysis of literature search as well as carefully and critically revising and approving the final manuscript.

\section{CONFLICT OF INTEREST}

Martin R., Moyal D. and Seité S. are employees of L'Oréal. Dréno B., Khammari A. and Henley J.B. have no interest to disclose.

\section{REFERENCES}

[1] E. A. Grice, J. A. Segre, Nat. Rev. Microbiol. 2011, 9, 244.

[2] E. A. Grice, H. H. Kong, S. Conlan, C. B. Deming, J. Davis, A. C. Young; NISC Comparative Sequencing Program, G. G. Bouffard, R. W. Blakesley, P. R. Murray, E. D. Green, M. L. Turner, J. A. Segre, Science 2009, 324, 1190.

[3] J. Di Domizio, A. Pagnoni, M. Huber, D. Hohl, M. Gilliet, Rev. Med. Suisse 2016, 12, 660.

[4] D. A. Sanchez, J. D. Nosanchuk, A. J. Friedman, J. Drugs Dermatol. 2015, 14, 127

[5] L. S. Weyrich, S. Dixit, A. G. Farrer, A. J. Friedman, Australas. J. Dermatol. 2015, 56, 268.

[6] K. Szabo, L. Erdei, B. S. Bolla, G. Tax, T. Biro, L. Kemeny, Br. J. Dermatol. 2016, doi: 10.1111/bjd.14967. [Epub ahead of print]

[7] S. Bellew, D. Thiboutot, J. Q. Del Rosso, J. Drugs Dermatol. 2011, 10, 582.

[8] S. Bhambri, J. Q. Del Rosso, A. Bhambri, J. Drugs Dermatol. 2009, 8, 615.

[9] J. Kim, M. T. Ochoa, S. R. Krutzik, O. Takeuchi, S. Uematsu, A. J. Legaspi, H. D. Brightbill, D. Holland, W. J. Cunliffe, S. Akira, P. A. Sieling, P. J. Godowski, R. L. Modlin, J. Immunol. 2002, 169, 1535.

[10] J. Kim, Dermatology 2005, 211, 193.

[11] F. Jasson, I. Nagy, A. C. Knol, et al., Exp. Dermatol. 2013, 22, 587.

[12] S. Fitz-Gibbon, S. Tomida, B. H. Chiu, L. Nguyen, C. Du, M. Liu, D. Elashoff, M. C. Erfe, A. Loncaric, J. Kim, R. L. Modlin, J. F. Miller, E. Sodergren, N. Craft, G. M. Weinstock, H. Li, J. Invest. Dermatol. 2013, 133, 2152.

[13] H. B. Lomholt, M. Kilian, PLoS ONE 2010, 5, e12277.

[14] D. Saint-Leger, A. Bague, E. Cohen, M. Chivot, Br. J. Dermatol. 1986, 114,535

[15] A. C. Logan, Arch. Dermatol. 2003, 139, 941; author reply 942-943.

[16] A. Bouslimani, C. Porto, C. M. Rath, M. Wang, Y. Guo, A. Gonzalez, D. Berg-Lyon, G. Ackermann, G. J. Moeller Christensen, T. Nakatsuji, L. Zhang, A. W. Borkowski, M. J. Meehan, K. Dorrestein, R. L. Gallo, N. Bandeira, R. Knight, T. Alexandrov, P. C. Dorrestein, Proc. Natl Acad. Sci. USA 2015, 112, E2120.

[17] Y. Belkaid, J. A. Segre, Science 2014, 346, 954.

[18] B. Ladizinski, R. McLean, K. C. Lee, D. J. Elpern, L. Eron, Int. J. Dermatol. 2014, 53, 1177.

[19] J. Peterson, S. Garges, M. Giovanni, P. Mclnnes, L. Wang, J. A. Schloss, V. Bonazzi, J. E. McEwen, K. A. Wetterstrand, C. Deal, C. C. Baker, V. Di Francesco, T. K. Howcroft, R. W. Karp, R. D. Lunsford, C. R. Wellington, T. Belachew, M. Wright, C. Giblin, H. David, M. Mills, R. Salomon, C. Mullins, B. Akolkar, L. Begg, C. Davis, L. Grandison, M. Humble, J. Khalsa, A. R. Little, H. Peavy, C. Pontzer, M. Portnoy, M. H. Sayre, P. Starke-Reed, S. Zakhari, J. Read, B. Watson, M. Guyer, Genome Res. 2009, 19, 2317.

[20] G. J. Christensen, C. F. Scholz, J. Enghild, H. Rohde, M. Kilian, A. Thürmer, E. Brzuszkiewicz, H. B. Lomholt, H. Brüggemann, BMC Genom. 2016, 17, 152.

[21] Y. Wang, S. Kuo, M. Shu, J. Yu, S. Huang, A. Dai, A. Two, R. L. Gallo, C. M. Huang, Appl. Microbiol. Biotechnol. 2014, 98, 411.

[22] B. Dreno, F. Poli, H. Pawin, C. Beylot, M. Faure, M. Chivot, N. Auffret, D. Moyse, F. Ballanger, J. Revuz, J. Eur. Acad. Dermatol. Venereol. 2011, 25, 43.

[23] J. B. Emerson, B. C. Thomas, W. Alvarez, J. F. Banfield, Environ. Microbiol. 2016, 18, 1686.

[24] J. G. Caporaso, C. L. Lauber, W. A. Walters, D. Berg-Lyons, C. A. Lozupone, P. J. Turnbaugh, N. Fierer, R. Knight, Proc. Natl Acad. Sci. USA 2011, 108(Suppl. 1), 4516.
[25] R. C. Edgar, Nat. Methods 2013, 10, 996.

[26] R. C. Edgar, Bioinformatics 2010, 26, 2460.

[27] Q. Wang, G. M. Garrity, J. M. Tiedje, J. R. Cole, Appl. Environ. Microbiol. 2007, 73, 5261.

[28] E. A. Grice, Semin. Cutan. Med. Surg. 2014, 33, 98.

[29] M. Wilson, Microbial Inhabitants of Humans: Their Ecology and Role in Health and Disease, Cambridge University Press, Cambridge 2005.

[30] K. Findley, J. Oh, J. Yang, S. Conlan, C. Deming, J. A. Meyer, D. Schoenfeld, E. Nomicos, M. Park; NIH Intramural Sequencing Center Comparative Sequencing Program., H. H. Kong, J. A. Segre, Nature 2013, 498, 367.

[31] M. Bek-Thomsen, H. B. Lomholt, M. Kilian, J. Clin. Microbiol. 2008, 46, 3355.

[32] X. Xia, Z. Li, K. Liu, Y. Wu, D. Jiang, Y. Lai, J. Invest. Dermatol. 2016, $136,621$.

[33] P. L. Zeeuwen, J. Boekhorst, E. H. van den Bogaard, H.D. de Koning, P.M. van de Kerkhof, D.M. Saulnier, I.I. van Swam, S.A. van Hijum, M. Kleerebezem, J. Schalkwijk, H.M. Timmerman, Genome Biol. 2012, 13, R101.

[34] S. Numata, H. Akamatsu, N. Akaza, A. Yagami, S. Nakata, K. Matsunaga, Dermatology 2014, 228, 86.

[35] E. A. Eady, A. M. Layton, J. H. Cove, Biomed. Res. Int. 2013, 2013, 679680.

[36] A. L. Zaenglein, A. L. Pathy, B. J. Schlosser, A. Alikhan, H. E. Baldwin, D. S. Berson, W. P. Bowe, E. M. Graber, J. C. Harper, S. Kang, J. E. Keri, J. J. Leyden, R. V. Reynolds, N. B. Silverberg, L. F. Stein Gold, M. M. Tollefson, J. S. Weiss, N. C. Dolan, A. A. Sagan, M. Stern, K. M. Boyer, R. Bhushan, J. Am. Acad. Dermatol. 2016, 74(), e933.

[37] Y. Fan, F. Hao, W. Wang, Y. Lu, L. He, G. Wang, W. Chen, J. Dermatol. 2016, 43, 406.

[38] K. Sardana, T. Gupta, B. Kumar, H. K. Gautam, V. K. Garg, Indian J. Dermatol. 2016, 61, 45.

[39] M. Juda, B. Chudzik-Rzad, A. Malm, Mem. Inst. Oswaldo Cruz 2016, $111,155$.

[40] D. P. Chikviladze, D. A. Metreveli, K. E. Gachechiladze, M. L. Mikeladze, Georgian Med. News 2012, 202, 60.

\section{SUPPORTING INFORMATION}

Additional Supporting Information may be found online in the supporting information tab for this article.

Table S1 Summary of patient demographics and clinical assessment of disease severity on Day 0.

Table S2 Lesion count and Shannon Index at Day $0(n=26)$.

Table S3 Main bacterial phyla and genus in percentage at the skin surface of the 3 sampled areas (comedones, papulo-pustular and skin area with no acne lesions for the hemi-face receiving Erythromycin $4 \%$ before (Day 0 ) and after (Day 28 ) treatment ( $n=26$ ).

Table S4 Main bacterial phyla and genus in percentage on the skin surface of the 3 sampled areas (comedones, papulo-pustular and skin area with no acne lesions for the hemi-face receiving the dermocosmetic before (Day 0) and after (Day 28) treatment ( $n=26$ ).

Fig. S1 Reduction in the number of papulo-pustular lesions and comedones on both hemi-faces after 28 days of treatment with either Erythromycin (A) or the dermocosmetic $(B) ;(n=26$, values are expressed in mean \pm SD).

How to cite this article: Dreno B, Martin R, Moyal D, Henley JB, Khammari A, Seité S. Skin microbiome and acne vulgaris: Staphylococcus, a new actor in acne. Exp Dermatol. 2017;26:798-803. https://doi.org/10.1111/exd.13296 